\title{
USO DE ESTERCO NO DESENVOLVIMENTO DE MUDAS DE MAMOEIRO COLONIZADAS COM FUNGOS MICORRíZICOS ${ }^{1}$
}

\author{
ALDO VILAR TRINDADE ${ }^{2}$, NELITA GONÇALVES FARIA ${ }^{3}$ e FLORÍCIO PINTO DE ALMEIDA ${ }^{4}$
}

\begin{abstract}
RESUMO - O trabalho objetivou a adequação de esterco na composição do substrato para produção de mudas de mamoeiro (Carica papaya L.), que permita o melhor estabelecimento da simbiose micorrízica e sua expressão no desenvolvimento da muda. Foram testadas cinco doses de esterco no substrato ( 0 , 5, 10, 20 e 30\%) e a inoculação ou não de Glomus etunicatum. Após 50 dias de cultivo concluiu-se que: o esterco promove máximo desenvolvimento das mudas nas doses de $20 \%$ e $30 \%$; a adição de $\mathrm{P}$ e K não resulta em benefícios adicionais no crescimento das mudas; a inoculação de G. etunicatum é eficiente para o desenvolvimento da muda até a dose de $10 \%$ de esterco; o fungo coloniza as mudas em doses de até $30 \%$ de esterco, mas com tendência decrescente; a combinação de $10 \%$ de esterco e inoculação do fungo micorrízico arbuscular promove a formação de plantas sadias, sem sintomas de deficiência nutricional e apropriadas para o transplantio ao campo.
\end{abstract}

Termos para indexação: Carica papaya, fumigação, micorriza, simbiose, substrato de cultivo

\author{
USE OF MANURE FOR DEVELOPMENT OF PAPAYA SEEDLINGS \\ COLONIZED WITH MYCORRHIZAL FUNGI
}

\begin{abstract}
This research tested the use of manure as part of the substrate for production of papaya (Carica papaya L.) seedlings enabling the establishment and expression of mycorrhizal symbiosis. Five levels of manure were tested: $0,5,10,20$ and $30 \%$ with or without inoculation of Glomus etunicatum. Results obtained after 50 days of culture showed that plants have higher growth at $20 \%$ and $30 \%$ of manure in the soil; use of $\mathrm{P}$ and $\mathrm{K}$ have no addition effect on plant growth; inoculation of G. etunicatum is efficient for development of plants in soils with up to $10 \%$ of manure; all levels of manure used permit the establishment of the fungi on the roots but with a decreasing effect at the highest levels of manure; the combination of $10 \%$ of manure and inoculation of AM fungi promote healthy plants without symptoms of nutrient deficiency and suitable for transplanting to the field.

Index terms: Carica papaya, fumigation, mycorrhizae, symbiosis, growing media.
\end{abstract}

\section{INTRODUÇÃO}

O mamoeiro apresenta elevada resposta à micorrização (Silva \& Siqueira, 1991; Auler, 1995; Trindade, 1998), porém, é uma cultura em cujo sistema de

\footnotetext{
${ }^{1}$ Aceito para publicação em 26 de novembro de 1999.

${ }^{2}$ Eng. Agrôn. D.Sc., Embrapa-Centro Nacional de Pesquisa de Mandioca e Fruticultura (CNPMF), Caixa Postal 007, CEP 44380-000 Cruz das Almas, BA.

E-mail: aldo@cnpmf.embrapa.br

${ }^{3}$ Eng. Agrôn., M.Sc., Universidade de Tocantins (UNITINS),CEP 77402-970 Gurupi, TO.

E-mail: unitins.gurupi@naves.com.br

${ }^{4}$ Eng. Agrôn., aluno do curso de mestrado, Universidade Federal da Paraíba (UFPB), CEP 58102-970 Campina Grande, PB. E-mail: floricio@deag.ufpb.br
}

produção é comum o emprego de doses maciças de adubação, especialmente de $\mathrm{P}$, que, em muitos casos, é aplicado mensalmente. Assim, vem sendo investigada a possibilidade de se produzir mudas já colonizadas com fungos micorrízicos arbusculares (FMA) competitivos e eficientes que venham a promover um bom desenvolvimento das plantas após o transplantio, e com isso promover economia de adubos, uma vez que as micorrizas proporcionam aumentos do volume de solo explorado pelas hifas e favorecem a absorção do P no momento em que é solubilizado ou liberado (Owusu-Bennoah \& Wild, 1980).

A micorrização de mudas de fruteiras tem sido indicada e várias são as espécies de FMA testadas, com o objetivo de melhoria da eficiência de absorção 
dos nutrientes pelas plantas e, conseqüentemente, redução dos gastos com fertilizantes (Silva \& Siqueira, 1991; Weber \& Amorim, 1994). O emprego de FMA constitui uma das alternativas para otimização da produção de mudas de mamoeiro (Auler, 1995). Entre os benefícios oriundos da micorrização, pode-se enfatizar o maior desenvolvimento das plantas, maior resistência à murcha causada por lesões na ação de transplante e estresse hídrico e proteção da planta ao ataque de fitopatógenos que atacam o sistema radicular (Gerdermann, 1975). A preocupação em aperfeiçoar as técnicas de produção de mudas é de suma importância, uma vez que o desenvolvimento inicial de mudas de mamoeiro tem relação direta com a precocidade e produção de frutos. Porém, é necessário que sejam estabelecidas as condições essenciais ao bom funcionamento da simbiose, para que o desempenho da micorriza seja assegurado.

As mudas normalmente são produzidas em substrato com potencial de inóculo baixo ou mesmo nulo, seja pelo uso de doses elevadas de adubos, seja pelo uso de fumigantes ou emprego de substratos inertes. $\mathrm{O}$ esterco constitui grande parte do substrato utilizado para produção das mudas, devendo interagir com a inoculação. Sendo um material orgânico, o esterco pode beneficiar a formação da associação. St. John et al. (1983) obtiveram evidências experimentais de que a matéria orgânica estimula o crescimento e a ramificação das hifas do fungo no solo, pela formação de um nicho fisiológico mais adequado para o crescimento do fungo. Ishac et al. (1986) relataram que o maior desenvolvimento radicular, promovido pela matéria orgânica, pode resultar em maior desenvolvimento de micorrizas na planta. A presença da matéria orgânica em substrato usado para a formação de mudas pode beneficiar o efeito da micorrização. Souza (1987), empregando uma dose de $30 \%$ de composto de esterco bovino mais palha de gramíneas, não obteve alteração na colonização micorrízica em plantas de café.

Por outro lado, algumas fontes de matéria orgânica podem afetar a colonização micorrízica. $O$ nível de fertilidade do substrato, principalmente quanto à disponibilidade de $\mathrm{P}$, é um dos principais fatores a ser determinado nos estudos da associação micorrízica, pois o P controla o grau de colonização das raízes pelos fungos endomicorrízicos. Como a reserva de nutrientes nos estercos é elevada, particularmente no que diz respeito ao $\mathrm{P}$, o uso de proporções elevadas no substrato de produção das mudas pode resultar em redução ou mesmo eliminação da colonização micorrízica (Trindade, 1992). Brechelt (1987) verificou que maiores quantidades de adubos orgânicos levaram à redução na colonização e eficiência micorrízica. Andrade (1991) verificou aumento na colonização de Brachiaria decumbens por FMA quando aplicou $25 \%$ de composto de lixo urbano em mistura com subsolo de área de mineração de ferro; entretanto, quando a dose foi de $50 \%$, a colonização foi significativamente reduzida.

Este estudo teve como objetivo verificar a proporção mais adequada de esterco no substrato, seus efeitos no estabelecimento da simbiose micorrízica e seu resultado no desenvolvimento da muda; também averiguou-se a necessidade de complemento com adubo solúvel.

\section{MATERIAL E MÉTODOS}

O experimento foi conduzido em casa de vegetação da Embrapa-Centro Nacional de Pesquisa de Mandioca e Fruticultura, em Cruz das Almas, BA, com início em 10 de outubro e prolongando-se até 25 de novembro de 1996.

Para composição do substrato de cultivo, utilizou-se um solo de textura franco-argilo-arenosa, fumigado com brometo de metila $\left(395 \mathrm{~mL} \mathrm{~m}^{-3}\right)$ e misturado com esterco bovino de curral maturado, parcialmente secado ao ar, e passado em peneira de $4 \mathrm{~mm}$ de malha (Tabela 1). O esterco apresentava $42,5 \%$ de umidade (com base em peso) e densidade de $0,55 \mathrm{~g} \mathrm{~cm}^{-3}$, quando da aplicação ao solo.

Os tratamentos foram compostos pelo uso de cinco proporções de esterco $(0,5,10,20$ e $30 \%$, volume/volume) (Tabela 1), e inoculação, ou não, do FMA Glomus etunicatum (fornecido pelo Departamento de Ciência do Solo da UFLA, Lavras, MG). Utilizou-se delineamento estatístico em blocos casualizados, com quatro repetições, sendo cada parcela composta por um recipiente (saco de plástico com capacidade para $1 \mathrm{dm}^{3}$ ). Foram testados ainda dois tratamentos adicionais, formados pela aplicação de esterco na proporção de $30 \%$, acrescido de $\mathrm{P}$, como superfosfato simples $\left(2 \mathrm{~kg} \mathrm{~m}^{-3}\right.$ de substrato) e $\mathrm{K}$, como cloreto de potássio $\left(0,5 \mathrm{~kg} \mathrm{~m}^{-3}\right.$ de substrato), em solo fumigado e não-fumigado.

As sementes de mamão (cv. Sunrise Solo) foram germinadas em areia + vermiculita $(1: 1, \mathrm{v} / \mathrm{v})$, efetuando-se a repicagem de uma plântula para cada recipiente de cultivo, 
quando apresentavam altura em torno de $5,0 \mathrm{~cm}$, e pelo menos um par de folhas definitivo. Na repicagem, efetivouse a inoculação, dispondo o inóculo misto (solo + areia, esporos, hifas, raízes de sorgo e micorrizas) em orifício central e logo em seguida a plântula. No tratamento-controle, não-inoculado, aplicou-se porção de substrato idêntico ao utilizado na produção de inóculo, contendo apenas raízes de sorgo. Durante a condução do experimento, foram aplicados $60 \mathrm{mg} \mathrm{dm}^{-3} \mathrm{de} \mathrm{N}$ em cobertura, via solução nutritiva, dividida em aplicações semanais.

Aos 20,30 e 45 dias após a repicagem, foram feitas medições de altura das mudas, e aos 50 dias, fez-se a coleta da parte aérea das plantas. Após secagem em estufa com ventilação forçada, a $70^{\circ} \mathrm{C}$, obteve-se o peso de matéria seca, e posteriormente fez-se determinação dos teores de $\mathrm{P}$ e N. Para avaliar a colonização micorrízica, fez-se uma amostra composta do sistema radicular de cada parcela, sendo, em seguida, submetida à clarificação e coloração (Phillips \& Hayman,1970) e a avaliação da presença do fungo, utilizando o método da placa reticulada, de acordo com metodologia descrita por Giovannetti \& Mosse (1980).
Os dados foram submetidos à analise de variância, com posterior aplicação de análise de regressão, e teste de Tukey, para comparação dos efeitos do esterco e da inoculação, respectivamente

\section{RESULTADOS E DISCUSSÃO}

Os resultados obtidos, relativos à utilização de esterco, confirmam informações já obtidas em estudos feitos com outras culturas perenes e semiperenes, obtendo-se aumentos no crescimento em altura e peso de matéria seca das mudas (Tabela 2), à medida que se aumenta a participação do esterco no substrato.

Os modelos de regressão ajustados (Tabela 3) revelam que a maior altura de planta foi atingida com doses inferiores à máxima aplicada, enquanto a produção de matéria seca foi maior com a dose de $30 \%$ de esterco.

TABELA 1. Análise química do solo, esterco e misturas, utilizados no experimento.

\begin{tabular}{|c|c|c|c|c|c|c|c|c|c|c|}
\hline Substrato $^{1}$ & $\mathrm{pH} \mathrm{em} \mathrm{H}_{2} \mathrm{O}$ & $\begin{array}{c}\mathrm{P} \\
--(\mathrm{mg} \\
\end{array}$ & $\begin{array}{c}\mathrm{K} \\
\left.\mathrm{dm}^{-3}\right)-- \\
\end{array}$ & $\mathrm{Ca}$ & $\mathrm{Mg}$ & $\begin{array}{c}\mathrm{Al} \\
\left(\mathrm{cmol}_{\mathrm{c}} \mathrm{dm}^{-3}\right)\end{array}$ & $\mathrm{Na}$ & CTC & $\begin{array}{c}\mathrm{V} \\
(\%) \\
\end{array}$ & $\begin{array}{c}\text { M.O. } \\
\left(\mathrm{g} \mathrm{dm}^{-3}\right)\end{array}$ \\
\hline Solo & 4,4 & 3 & 31 & 0,8 & 0,4 & 0,8 & 0,03 & 4,83 & 27,1 & 16,1 \\
\hline Esterco & 8,0 & 792 & 5673 & 9,4 & 10,4 & 0,0 & 3,30 & 37,60 & 100,0 & 239,0 \\
\hline Esterco $5 \%$ & 4,8 & 21 & 209 & 1,6 & 1,4 & 0,2 & 0,13 & 7,40 & 49,5 & 23,1 \\
\hline Esterco $10 \%$ & 5,5 & 41 & 374 & 2,3 & 1,5 & 0,0 & 0,21 & 8,05 & 61,7 & 25,1 \\
\hline Esterco $20 \%$ & 6,1 & 60 & 616 & 3,3 & 2,7 & 0,0 & 0,37 & 10,04 & 79,2 & 35,3 \\
\hline Esterco $30 \%$ & 6,6 & 90 & 1089 & 3,8 & 3,0 & 0,0 & 0,51 & 11,53 & 87,6 & 40,8 \\
\hline Esterco $30 \%+\mathrm{P}+\mathrm{K}(\mathrm{NF})$ & 6,2 & 264 & 2046 & 6,1 & 2,9 & 0,0 & 0,77 & 17,31 & 86,7 & 40,1 \\
\hline Esterco $30 \%+\mathrm{P}+\mathrm{K}(\mathrm{F})$ & 6,5 & 288 & 1386 & 6,0 & 3,2 & 0,0 & 0,77 & 14,94 & 90,4 & 47,6 \\
\hline
\end{tabular}

${ }^{1} \mathrm{NF}$ : solo não-fumigado; F: solo fumigado.

TABELA 2. Altura de plantas e produção de matéria seca da parte aérea de mudas de mamoeiro em função da aplicação de esterco, complementação mineral e inoculação de Glomus etunicatum ${ }^{1}$.

\begin{tabular}{|c|c|c|c|c|c|c|c|c|}
\hline \multirow{3}{*}{$\begin{array}{c}\text { Doses de } \\
\text { esterco }(\%)^{2}\end{array}$} & \multicolumn{6}{|c|}{ Altura $(\mathrm{cm})$} & \multirow{2}{*}{\multicolumn{2}{|c|}{$\begin{array}{c}\text { Matéria seca da parte } \\
\text { aérea (g/planta) }\end{array}$}} \\
\hline & \multicolumn{2}{|c|}{$20 \operatorname{dias}^{3}$} & \multicolumn{2}{|c|}{30 dias } & \multicolumn{2}{|c|}{45 dias } & & \\
\hline & Não-inoculado & Inoculado & Não-inoculado & $\overline{\text { Inoculado }}$ & Não-inoculado & Inoculado & Não-inoculado & Inoculado \\
\hline 0 & $3,9 \mathrm{a}$ & $4,4 \mathrm{a}$ & $4,6 \mathrm{a}$ & $5,7 \mathrm{a}$ & $4,6 \mathrm{a}$ & $7,0 \mathrm{a}$ & $0,02 \mathrm{a}$ & $0,09 a$ \\
\hline 5 & $4,5 \mathrm{a}$ & $5,6 a$ & $6,1 b$ & $10,1 \mathrm{a}$ & $7,1 \mathrm{~b}$ & $16,1 \mathrm{a}$ & $0,08 \mathrm{~b}$ & $0,61 \mathrm{a}$ \\
\hline 10 & $5,9 a$ & $5,5 \mathrm{a}$ & $9,6 a$ & $10,5 \mathrm{a}$ & $15,9 b$ & $18,0 \mathrm{a}$ & $0,42 b$ & $0,87 \mathrm{a}$ \\
\hline 20 & $6,7 \mathrm{a}$ & $5,3 \mathrm{a}$ & $13,5 \mathrm{a}$ & $11,9 \mathrm{a}$ & $21,5 \mathrm{a}$ & $19,5 \mathrm{a}$ & $1,14 \mathrm{a}$ & $0,90 \mathrm{a}$ \\
\hline 30 & $6,5 \mathrm{a}$ & $6,3 \mathrm{a}$ & $12,8 \mathrm{a}$ & $11,0 \mathrm{a}$ & $18,9 \mathrm{a}$ & $18,1 \mathrm{a}$ & $1,24 \mathrm{a}$ & $0,97 \mathrm{a}$ \\
\hline $30+\mathrm{P}+\mathrm{K}(\mathrm{NF})$ & 6,1 & - & 13,4 & - & 21,2 & - & 1,30 & \\
\hline $30+\mathrm{P}+\mathrm{K}(\mathrm{F})$ & 6,4 & - & 13,5 & - & 20,2 & - & 1,33 & \\
\hline
\end{tabular}

1 Valores seguidos da mesma letra minúscula nas linhas, não diferem estatisticamente a $5 \%$ de probabilidade, pelo teste de Tukey.

2 NF: solo não-fumigado; F: solo fumigado.

3 Dias após repicagem 
TABELA 3. Equações de regressão para altura de plantas e matéria seca da parte aérea de mamoeiro em função da aplicação de esterco e inoculação de Glomus etunicatum.

\begin{tabular}{llll}
\hline Característica & Inoculação & Regressão & $\mathrm{R}^{2}$ \\
\hline Altura - 20 dias & Não-inoculado & $\mathrm{Y}=3,73+0,251 \mathrm{X}-0,00527 \mathrm{X}^{2}$ & 0,97 \\
Altura - 20 dias & Inoculado & $\mathrm{Y}=4,49+0,386 \mathrm{X}^{1 / 2}-0,0203 \mathrm{X}$ & 0,74 \\
Altura - 30 dias & Não-inoculado & $\mathrm{Y}=3,91+0,713 \mathrm{X}-0,01359 \mathrm{X}^{2}$ & 0,96 \\
Altura - 30 dias & Inoculado & $\mathrm{Y}=5,68+2,539 \mathrm{X}^{1 / 2}-0,28 \mathrm{X}$ & 0,98 \\
Altura - 45 dias & Não-inoculado & $\mathrm{Y}=3,09+1,53 \mathrm{X}-0,0329 \mathrm{X}^{2}$ & 0,95 \\
Altura - 45 dias & Inoculado & $\mathrm{Y}=6,95+5,55 \mathrm{X}^{1 / 2}-0,635 \mathrm{X}$ & 0,99 \\
Parte aérea & Não-inoculado & $\mathrm{Y}=0,0246+0,46396 \mathrm{X}$ & 0,93 \\
Parte aérea & Inoculado & $\mathrm{Y}=0,086+0,319 \mathrm{X}^{1 / 2}-0,02917 \mathrm{X}$ & 0,98 \\
\hline
\end{tabular}

Os tratamentos adicionais permitiram observar que a suplementação de $\mathrm{P}$ e $\mathrm{K}$ ao substrato contendo $30 \%$ de esterco não teve efeito significativo no desenvolvimento das mudas (Tabela 2). Com relação à inoculação do fungo micorrízico, observou-se que o melhor efeito ocorreu em dose de esterco inferior às doses de $20 \%$ e $30 \%$.

O FMA Glomus etunicatum foi eficiente nas doses de $5 \%$ e $10 \%$ de esterco, tendo proporcionado mudas com maior altura e acúmulo de massa vegetal (Tabela 2), confirmado pelo ajuste do modelo raizquadrático, o qual reflete incrementos maiores com doses menores.

O efeito da inoculação foi maior com o avanço da idade das mudas (Tabela 2). Levando-se em consideração que o esterco de gado apresenta composição variável em razão da sua origem, o resultado neste trabalho difere do obtido por Silveira et al. (1996), em que a inoculação de fungos micorrízicos beneficiou o desenvolvimento de mudas de maracujá mesmo em substrato composto de até $40 \%$ de esterco. Nas demais proporções de esterco, a associação micorrízica não beneficiou o desenvolvimento da muda, e o fungo causou um efeito depressivo, que pode ocorrer quando o substrato apresenta elevados níveis de $\mathrm{P}$, atingindo uma zona de simbiose parasítica, segundo modelo de Siqueira \& Colozzi Filho (1986). Embora o maior acúmulo de matéria vegetal ao final do experimento tenha-se dado nas maiores proporções de esterco, o uso de $10 \%$ do resíduo em associação à inoculação resultou em mudas bem formadas (Tabela 2).

Os teores de $\mathrm{P}$ na parte aérea foram superiores nas plantas infectadas, particularmente nas doses mais baixas de esterco, onde ocorreram os maiores efeitos do fungo no crescimento das plantas (Tabela 4). A presença do fungo micorrízico aumen- tou em $80 \%$ o $\mathrm{P}$ absorvido pelas plantas na dose de $5 \%$ de esterco. Na dose de $10 \%$ de esterco, o aumento do $\mathrm{P}$ absorvido, com a inoculação, foi de $53 \%$, o que corrobora o papel do FMA no aumento da zona de exploração das raízes. Este efeito é mais pronunciado quando os teores de $\mathrm{P}$ no substrato são mais baixos. Quanto aos teores de $\mathrm{N}$, aparentemente não houve efeito da inoculação. Como não foi possível analisar os teores desse nutriente no tratamento sem adição de esterco (em decorrência da pequena quantidade da amostra), não se sabe até que ponto o resíduo contribuiu para a nutrição nitrogenada. Quanto a esse aspecto, os dados mostram que não houve aumento na absorção do $\mathrm{N}$ pelas mudas, em proporções de esterco além do 5\% adicionado (Tabela 4). Com o uso de suplementação de P e K no substrato contendo $30 \%$ de esterco, observou-se um consumo de luxo de $\mathrm{P}$ por parte das plantas, já que houve um aumento nos teores do nutriente na parte aérea sem uma conversão em massa vegetal significativa.

Por ocasião da coleta, foi verificado que o sistema radicular pouco se estendeu no volume do substrato, e que houve uma concentração na metade superior do recipiente. Como se trata de uma planta que apresenta elevada taxa de crescimento de parte aérea, ressalta-se a necessidade da associação micorrízica ou de uma adubação mais elevada para o seu crescimento ideal, considerando-se o seu pequeno suporte radicular. De acordo com Koide (1991), a capacidade de produção de raízes pela planta é um dos principais fatores que determinam sua dependência à micorriza, o que foi comprovado quanto ao mamoeiro, por Trindade (1998), quando verificou que variedades com menor comprimento da raiz respondem mais à inoculação de FMA.

A inoculação promoveu colonização micorrízica elevada até a dose de $20 \%$ de esterco (Fig. 1). Com a 
TABELA 4. Nitrogênio e fósforo absorvidos pelas mudas de mamoeiro em função da aplicação de esterco, complementação mineral e inoculação de Glomus etunicatum.

\begin{tabular}{|c|c|c|c|c|}
\hline \multirow[t]{2}{*}{ Doses de esterco $(\%)^{1}$} & \multicolumn{2}{|c|}{ Nitrogênio $(\mathrm{g} / \mathrm{kg})$} & \multicolumn{2}{|c|}{ Fósforo (g/kg) } \\
\hline & Não-inoculado & Inoculado & Não-inoculado & Inoculado \\
\hline 0 & n.d. ${ }^{2}$ & n.d. & n.d. & n.d. \\
\hline 5 & n.d. & 34,2 & 1,5 & 2,7 \\
\hline 10 & 33,3 & 31,2 & 1,9 & 2,9 \\
\hline 20 & 29,4 & 35,8 & 2,3 & 2,8 \\
\hline 30 & 31,9 & 30,2 & 3,2 & 3,0 \\
\hline $30+\mathrm{P}+\mathrm{K}(\mathrm{NF})$ & 28,0 & - & 5,7 & - \\
\hline $30+\mathrm{P}+\mathrm{K}(\mathrm{F})$ & 31,9 & - & 5,8 & - \\
\hline
\end{tabular}

$1 \mathrm{NF}$ : solo não-fumigado; F: solo fumigado.

2 Não determinado.

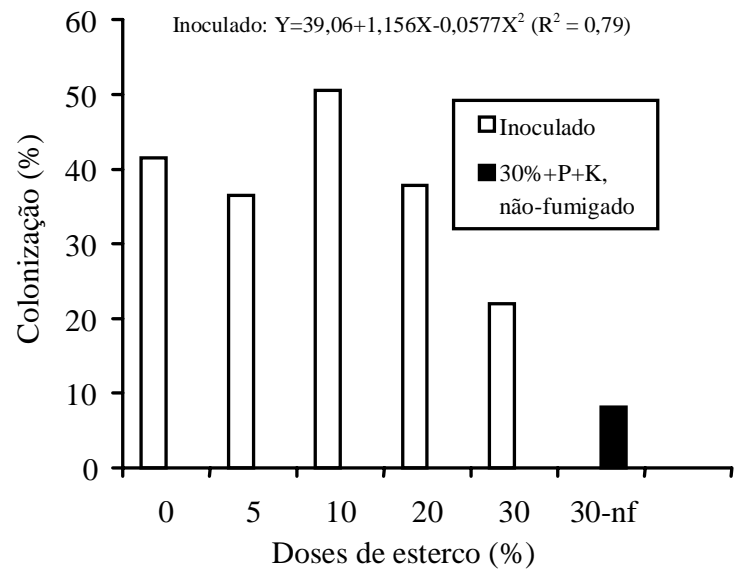

FIG. 1. Colonização micorrízica de plantas de mamoeiro aos 50 dias após repicagem, em função da aplicação de esterco, complementação mineral e inoculação de Glomus etunicatum.

aplicação de $30 \%$ de esterco, houve redução significativa na colonização, mas esta permaneceu em valores acima de $10 \%$. Esses valores são maiores do que os verificados na colonização pelos fungos nativos (solo não-fumigado em $30 \%+\mathrm{P}+\mathrm{K}$ ) no tratamento adicional. Também são maiores do que os verificados por Trintade (1998), em mudas de mamão, produzidas em viveiro comercial, onde é comum a utilização de substratos à base de $1 / 3$ de esterco. Isso sugere que diante de um amplo trabalho de seleção de FMA para a cultura é possível obter uma espécie ou um isolado capaz de formar colonização expressiva na raiz com doses de esterco dessa magni- tude, e que, em última análise, permita a introdução de um fungo que venha a promover benefícios em condições de campo.

\section{CONCLUSÕES}

1. O esterco promove máximo desenvolvimento das mudas nas doses de $20 \%$ e $30 \%$.

2. A adição de $\mathrm{P}$ e $\mathrm{K}$ não resulta em benefícios adicionais no crescimento das mudas de mamoeiro.

3. A inoculação de Glomus etunicatum é eficiente para o desenvolvimento da muda até a dose de $10 \%$ de esterco.

4. O fungo coloniza as mudas em doses de até $30 \%$ de esterco, mas com tendência decrescente

5. A combinação da aplicação de $10 \%$ de esterco e inoculação do fungo micorrízico arbuscular promove a formação de mudas sadias e apropriadas ao transplantio para o campo.

\section{REFERÊNCIAS}

ANDRADE, L.A.B.L. Influência da associação micorrízica e da adição de matéria orgânica sobre o crescimento de Brachiaria decumbens em estéril de mineração de ferro e bauxita. Viçosa : UFV, 1991. 53p. Dissertação de Mestrado.

AULER, P.A.M. Desenvolvimento inicial do mamoeiro (Carica papaya L.) relacionado à disponibilidade de $\mathbf{P}$ no solo e à colonização pelo fungo MA 
Glomus macrocarpum. Botucatu : UNESP-FCA, 1995. 94p. Dissertação de Mestrado.

BRECHELT, A. Wirkung verschiedener organischer Düngemittel auf die Effizienz der VA-Mykorrhiza. Journal of Agronomy and Crop Science, Berlim, v. 158, p.280-286, 1987.

GERDEMANN, I.W. Vesicular-arbuscular mycorrhizal. In: TORREY, J.G.; CLARKSON, D.T. (Ed.). The development and function of roots. London Academic, 1975. p.575-591.

GIOVANNETTI, M.; MOSSE, B. An evaluation of techniques for measuring vesicular-arbuscular mycorrhizal infection in roots. New Phytologist, Cambridge, Grã-Bretanha, v.84, n.3, p.482-500, 1980.

ISHAC, Y.Z.; EL-HADDAD, M.E.; DAFT, M.J.; RAMADAN, E.M.; ELDEMERDASH, M.E. Effect of seed inoculation, mycorrhizal infection and organic amendment on wheat growth. Plant and Soil, Dordrecht, v.90, p.373-382, 1986.

KOIDE, R.T. Nutrient supply, nutrient demand and plant response to mycorrhizal infection. New Phytologist, Cambridge, Grã-Bretanha, v.117, n.2, p.365-386, 1991.

OWUSU-BENNOAH, E.; WILD,A. Effects of vesiculararbuscular mycorrhiza on the size of the labile pool of soil phosphate. Plant and Soil, Dordrecht, v.54, n. 2, p. $233-242,1980$

PHILLIPS, J.M.; HAYMAN, D.S. Improved procedures for clearing roots and staining parasitic and vesiculararbuscular mycorrhizal fungi for rapid assessment of infection. British Mycological Society Transactions, Cambridge, Grã-Bretanha, v.55, p.158-161, 1970.

SILVA, L.F.C.; SIQUEIRA, J.O. Crescimento e teores de nutrientes de mudas de abacateiro, mangueira e mamoeiro sob influência de diferentes espécies de fungos micorrízicos vesículo-arbusculares. Revista Brasileira de Ciência do Solo, Campinas, v.15, n.3, p.283-288, 1991.
SILVEIRA, A.P.D.; AZEVEDO, I.C.; OLIVEIRA, E.; MELETTI, L.M.M. Crescimento de plantas micorrizadas de maracujazeiro sob influência da adubação fosfática e da matéria orgânica. In: CONGRESSO LATINO-AMERICANO DE CIÊNCIA DO SOLO, 13., 1996, Águas de Lindóia. Anais. Águas de Lindóia : Sociedade Latino-Americana de Ciência do Solo, 1996. CD-ROM. Seção trabalhos voluntários.

SIQUEIRA, J.; COLOZZI FILHO, A. Micorrizas vesículo-arbusculares em mudas de cafeeiro. II. Efeito do $\mathrm{P}$ no estabelecimento e funcionamento da simbiose. Revista Brasileira de Ciência do Solo, Campinas, v.10, n.3, p.207-211, 1986.

SOUZA, C.S. Desenvolvimento de mudas de cafeeiro (Coffea arabica L.) inoculadas com Gigaspora margarita (Becker \& Hall) em substrato com e sem matéria orgânica e diferentes doses de superfosfato simples. Lavras : ESAL, 1987. 237p. Dissertação de Mestrado.

ST. JOHN, T.V.; COLEMAN, D.C.; REID, C.P.P. Association of vesicular-arbuscular mycorrhizal hyphae with soil organic particles. Ecology, Washington, v.64, p.957-959, 1983

TRINDADE, A.V. Crescimento e composição mineral de mudas de Eucalyptus grandis em resposta à inoculação com fungos micorrízicos vesículoarbusculares e à aplicação de composto orgânico. Viçosa : UFV, 1992. 84p. Dissertação de Mestrado.

TRINDADE, A.V. Micorrizas arbusculares em mamoeiro. Lavras : UFLA, 1998. 178p. Tese de Doutorado.

WEBER, O.B.;AMORIM, S.M.C. de. Adubação fosfática e inoculação de fungos micorrízicos vesículoarbusculares em mamoeiro 'Solo'. Revista Brasileira de Ciência do Solo, Campinas, v.18, n.2, p.187-191, 1994. 